\title{
On the Singularities of Solutions of Differential Equations of the Elliptic Type*
}

\author{
E. NETANYAHU
}

Stanford University, Stanford, California

Communicated by S. BERGMAN

1. Introduction. We consider partial differential equations of the elliptic type

$$
\mathbf{L}(u) \equiv \frac{1}{4} \Delta u+\frac{1}{2} A u_{x}+\frac{1}{2} B u_{y}+C u=0
$$

where $A, B, C$ are assumed to be entire functions of $x$ and $y$ (even when continued to complex values).

In the present paper we show that subsequences $\left\{D_{m n}\right\}, n$ fixed, $m=0,1,2, \cdots$ of the series development of the real solution

$$
U\left(z, z^{*}\right)=\sum_{m, n=0}^{\infty} D_{m n} z^{m} z^{*^{n}}
$$

of (1.1) determine the location and character of the singularities of $U\left(z, z^{*}\right)$ up to a finite number of isolated ones.

Here $z=x+i y, z^{*}=x-i y, D_{m n}=\vec{D}_{n m}$.

We conclude with some remarks about properties of subsequences of coefficients of the developments of $U\left(z, z^{*}\right)$ around different centers.

We mention that the results obtained are independent of the coefficients $A, B, C$ of (1.1).

2. The method of the integral operator. Some methods for the study of harmonic functions of two variables are based on their connection with analytic

* This research was supported by the United States Air Force through the Office of Scientific Research of the Research and Development Command. Contract No. AF 18(600)1036 . 
functions of one complex variable. Generalizing this procedure to the case of the linear partial differential equation (1.1), Bergman (see [1] and [3]) introduced the integral operator method. We give here a brief survey of this method and mention some of the results which we need in the sequel.

In complex notation*

$$
\begin{aligned}
z & =x+i y, \quad z^{*}=x-i y \\
\frac{\partial}{\partial z} & =\frac{1}{2}\left(\frac{\partial}{\partial x}-i \frac{\partial}{\partial y}\right), \quad \frac{\partial}{\partial z^{*}}=\frac{1}{2}\left(\frac{\partial}{\partial x}+i \frac{\partial}{\partial y}\right),
\end{aligned}
$$

the differential equation (1.1) assumes the form

$$
u_{z z^{*}}+a u_{z}+a^{*} u_{z^{*}}+c u=0,
$$

where

$$
\begin{aligned}
a & \equiv a\left(z, z^{*}\right)=\frac{1}{2}\left[A\left(\frac{z+z^{*}}{2}, \frac{z-z^{*}}{2 i}\right)+i B\left(\frac{z+z^{*}}{2}, \frac{z-z^{*}}{2 i}\right)\right], \\
a^{*} & \equiv \bar{a}\left(z^{*}, z\right), c \equiv c\left(z, z^{*}\right)=C\left(\frac{z+z^{*}}{2}, \frac{z-z^{*}}{2 i}\right)
\end{aligned}
$$

On the assumption that $A, B, C$ are entire functions of $x$ and $y$ it follows that $a\left(z, z^{*}\right)$ and $c\left(z, z^{*}\right)$ are entire functions of $z$ and $z^{*}$ and can be written in the form of power series

$$
\begin{aligned}
& a\left(z, z^{*}\right)=\sum_{m, n=0}^{\infty} a_{m n} z^{m} z^{* n}=\sum_{n=0}^{\infty} a_{n}(z) z^{* n} . \\
& c\left(z, z^{*}\right)=\sum_{m, n=0}^{\infty} c_{m n} z^{m} z^{* n}=\sum_{n=0}^{\infty} c_{n}(z) y^{* n} .
\end{aligned}
$$

In [1] and [2] Bergman associates with every real solution $U\left(z, z^{*}\right)$ of (1.1) an analytic function $g(z)$ of one complex variable. By means of the integral operator** this function is transformed into a complex solution $u\left(z, z^{*}\right)$ of $(2.3)$ whose real part is $U\left(z, z^{*}\right)$. The relations between $u\left(z, z^{*}\right)$ and $U\left(z, z^{*}\right)$ are similar in many respects to those between an analytic function of one complex variable and its real part.

As Bergman showed (see [1])

$$
U\left(z, z^{*}\right)=\frac{1}{2}\left[\mathbf{p}(g(z))+\mathbf{p}^{*}\left(\bar{g}\left(z^{*}\right)\right)\right]
$$

\footnotetext{
$* z^{*}$ becomes $\bar{z}$ when $x$ and $y$ are real.

** The integral operator used in this paper is denoted by BERGMAN as "integral opera. tor of the first kind". Since we use only this kind of operator we omit "the first kind".
} 
where

$$
\begin{aligned}
\mathbf{p}(g(z)) & =u\left(z, z^{*}\right) \\
& =R\left(z, z^{*}\right)\left[g(z)+\sum_{n=1}^{\infty} \frac{(2 n) !}{2^{2 n} n !} Q^{(n)}\left(z, z^{*}\right) \int_{0}^{z} \cdots \int_{0}^{z_{n-1}} g\left(z_{n}\right) d z_{n} \cdots d z_{1}\right] \\
& =R\left(z, z^{*}\right)\left[g(z)+\sum_{n=1}^{\infty} \frac{Q^{(n)}\left(z, z^{*}\right)}{2^{2 n}} \frac{B(n, n+1)}{\left.B(z-\zeta)^{n-1} g(\zeta) d \zeta\right],}\right. \\
R\left(z, z^{*}\right) & =\exp \left[-\int_{0}^{z^{*}} a\left(z, z^{*}\right) d z^{*}\right] .
\end{aligned}
$$

$\mathbf{p}^{*}$ is an operator formed from $\vec{R}$ and $\overline{\mathrm{Q}^{(n)}}$ in just the same way as $\mathbf{p}$ is formed from $R$ and $Q^{(n)}$.

The functions $R\left(z, z^{*}\right)$ and $Q^{(n)}\left(z, z^{*}\right)$ depend only on the equation (2.3) and satisfy

$$
R(z, 0)=1, Q^{(n)}(z, 0)=0, n=1,2, \cdots .
$$

By continuing our solutions to complex arguments and considering them in the plane $z^{*}=0$ we obtain the formula

$$
U(z, 0)=\frac{1}{2}\left[g(z)+R^{*}(0, z) g^{*}(0)\right] .
$$

Remark. In the case of Laplace's equation every solution $H\left(z, z^{*}\right)$ can be written in the form

$$
H\left(z, z^{*}\right)=\frac{1}{2}\left(g(z)+g^{*}\left(z^{*}\right)\right)
$$

where $g(z)$ is an analytic function. If we continue the solution so that $z$ and $z^{*}$ are independent complex variables and then consider the solution in the characteristic plane $z^{*}=0$ we obtain the relation

$$
H(z, 0)=\frac{1}{2}\left[g(z)+g^{*}(0)\right] .
$$

In analogy to Laplace's equation we have to prescribe the value $g^{*}(0)$ in order to determine $g(z)$ uniquely from (2.7).

If the series development of the solution $u\left(z, z^{*}\right)$ of $(2.3)$.is

$$
u\left(z, z^{*}\right)=\sum_{m, n=0}^{\infty} u_{m n} z^{m} z^{*^{n}}
$$

then we get from $(2.6)$

$$
u(z, 0)=\sum_{m=0}^{\infty} u_{m 0} z^{m}=g(z)=\sum_{m=0}^{\infty} g_{m} z^{m},
$$

or

$$
u_{m 0}=g_{m} .
$$


From this and the fact that infinite sums in (2.6) converge in every simply connected domain where $g(z)$ is regular, it follows that the subsequence $\left\{u_{m 0}\right\}$ determines the domain of regularity of the complex solution $u\left(z, z^{*}\right)$. From (2.7) it is seen that the domain of regularity of the real solution is also determined by this subsequence (see [3]).

Therefore the subsequence $\left\{D_{n 0}\right\}, n=0,1,2, \cdots$, determines the domain of regularity of $U\left(z, z^{*}\right)$ independently of the coefficients $A, B, C$ of (1.1). In the following section we obtain a result which to some extent is analogous to the above mentioned relation in the case of the subsequence $\left\{D_{n k}\right\}$ for $k>0$.

3. Relations between the subsequences $\left\{D_{n k}\right\}, k$ fixed, $n=0,1,2, \cdots$, and the domain of regularity of $U\left(z, z^{*}\right)$. For convenience we introduce the following notations:

$$
\begin{aligned}
u\left(z, z^{*}\right) & =\sum_{k=0}^{\infty} u_{k}(z) z^{* k} & =\sum_{k=0}^{\infty} v_{k}\left(z^{*}\right) z^{k} \\
u_{k}(z) & =\sum_{m=0}^{\infty} u_{m k} z^{m}, v_{k}\left(z^{*}\right) & =\sum_{m=0}^{\infty} u_{k m} z^{* m} \\
a\left(z, z^{*}\right) & =\sum_{k=0}^{\infty} a_{k}\left(z^{*}\right) z^{k} & =\sum_{k=0}^{\infty} d_{k}(z) z^{* k} \\
a^{*}\left(z^{*}, z\right) & =\sum_{k=0}^{\infty} b_{k}\left(z^{*}\right) z^{k} & =\sum_{k=0}^{\infty} e_{k}(z) z^{* k} \\
c\left(z, z^{*}\right) & =\sum_{k=0}^{\infty} c_{k}\left(z^{*}\right) z^{k} & =\sum_{k=0}^{\infty} f_{k}(z) z^{* k}
\end{aligned}
$$

where the functions $a_{k}, b_{k}, c_{k}, d_{k}, e_{k}, f_{k}$ are entire functions of their arguments.

We first prove the following two lemmas:

Lemma 1. In the complex solution

$$
p(g(z))=u\left(z, z^{*}\right)=\sum_{k=0}^{\infty} v_{k}\left(z^{*}\right) z^{k}
$$

of (2.3) the functions $v_{k}\left(z^{*}\right), k=0,1,2, \cdots$, are entire functions.

Proof. By introducing $u\left(z, z^{*}\right)$ into (2.3) and equating to zero the coefficients of the powers of $z$ and taking into consideration (3.1), we get the following system of linear differential equations:

$$
\begin{aligned}
& \begin{aligned}
& v_{1}^{\prime}\left(z^{*}\right)+a_{0}\left(z^{*}\right) v_{1}\left(z^{*}\right)=0 \\
& 2 v_{2}^{\prime}\left(z^{*}\right)+2 a_{0}\left(z^{*}\right) v_{2}\left(z^{*}\right)+b_{0}\left(z^{*}\right) v_{1}^{\prime}\left(z^{*}\right)+ {\left[a_{1}\left(z^{*}\right)+c_{0}\left(z^{*}\right)\right] v_{1}\left(z^{*}\right) } \\
&+b_{1}\left(z^{*}\right) v_{0}^{\prime}\left(z^{*}\right)+c_{1}\left(z^{*}\right) v_{0}\left(z^{*}\right)=0 \\
& 3 v_{3}^{\prime}\left(z^{*}\right)+3 a_{0}\left(z^{*}\right) v_{3}\left(z^{*}\right)+b_{0}\left(z^{*}\right) v_{2}^{\prime}\left(z^{*}\right)+ {\left[2 a_{0}\left(z^{*}\right)+c_{0}\left(z^{*}\right)\right] v_{2}\left(z^{*}\right) } \\
&+b_{1}\left(z^{*}\right) a_{1}^{\prime}\left(z^{*}\right)+\left[a_{2}\left(z^{*}\right)+c_{1}\left(z^{*}\right)\right] v_{1}\left(z^{*}\right)+b_{2}\left(z^{*}\right) v_{0}^{\prime}\left(z^{*}\right)+c_{2}\left(z^{*}\right) v_{0}\left(z^{*}\right)=0
\end{aligned}
\end{aligned}
$$


From (2.6) follows that

$$
v_{0}(z)=u\left(0, z^{*}\right)=R\left(0, z^{*}\right) g(0)=g(0) \exp \left[-\int_{0}^{z^{*}} a\left(0, z^{*}\right) d z^{*}\right]
$$

is an entire function. Hence, on the assumption that the functions $a_{k}\left(z^{*}\right), b_{k}\left(z^{*}\right)$, $c_{k}\left(z^{*}\right)$ are entire functions, we conclude from (3.2) that all the functions $v_{k}\left(z^{*}\right)$ are entire, which proves the lemma.

Lemma 2. If $u_{k}(z), k>0$, is not an entire function, then in every simply connected domain $D, 0 \varepsilon D$, in which $u_{k}(z)$ is regular, the complex solution $u\left(z, z^{*}\right)=$ $\sum_{k=0}^{\infty} u_{k}(z) z^{* k}$ of (2.3) has, at most, finitely many isolated singularities.

Proof. Again, by introducing $u\left(z, z^{*}\right)$ in (2.3) and equating to zero the coefficients of the powers of $z^{*}$ we obtain

$$
\begin{aligned}
& u_{1}^{\prime}(z)+e_{0}(z) u_{1}(z)+ d_{0}(z) u_{0}^{\prime}(z)+f_{0}(z) u_{0}(z)=0 \\
& 2 u_{2}^{\prime}(z)+2 e_{0}(z) u_{2}(z)+ d_{0}(z) u_{1}^{\prime}(z)+\left[e_{1}(z)+f_{0}(z)\right] u_{1}(z)+d_{1}(z) u_{0}^{\prime}(z) \\
&+f_{1}(z) u_{0}(z)=0 \\
& 3 u_{3}^{\prime}(z)+3 e_{0}(z) u_{3}(z)+ d_{0}(z) u_{2}^{\prime}(z)+\left[2 e_{1}(z)+f_{0}(z)\right] u_{2}(z)+d_{1}(z) u_{1}^{\prime}(z) \\
&+\left[e_{2}(z)+f_{1}(z)\right] u_{1}(z)+d_{2}(z) u_{0}^{\prime}(z)+f_{2}(z) u_{0}(z)=0
\end{aligned}
$$

If now $u_{k}(z), k>0$ fixed, is not an entire function, its singularities come from an expression which is a linear combination of $u_{k-1}^{\prime}(z), u_{k-1}(z), \cdots, u_{0}^{\prime}(z), u_{0}(z)$ with coefficients which are entire functions. By induction it follows that the only singularities of $u_{k}(z)$ are singularities of $u_{0}(z)$, some of which may be cancelled.

From the theorem proved by Bergman [3] that $u\left(z, z^{*}\right)$ is regular in every simply connected domain (containing the origin) which is the product of $\left[z^{*}<\infty\right]$ and the domain of regularity of $u_{0}(z)$, the proof of the lemma follows.

By the aid of lemmas 1 and 2 we prove the following

Theorem. Let

$$
U(z, \bar{z})=\sum_{m, k=0}^{\infty} D_{m k} z^{m} \bar{z}^{k}=\sum_{k=0}^{\infty} U_{k}(z) \bar{z}^{k}, \quad D_{m k}=\bar{D}_{k m}
$$

be a real solution (1.1).

If $U_{k}(z), k>0$, is not an entire function then in every simply connected domain $D, 0 \varepsilon D$, in which $U_{k}(z)$ is regular, $U(z, \bar{z})$ has, at most, finitely many isolated singularities.

Proof. We have

$$
U(z, \bar{z})=\frac{1}{2} \sum_{m, k=0}^{\infty}\left(u_{m k}+\bar{u}_{k m}\right) z^{m} \bar{z}^{k}
$$


i.e.

$$
D_{m k}=\frac{1}{2}\left[u_{m k}+\bar{u}_{k m}\right]
$$

Hence

$$
\sum_{m=0}^{\infty} D_{m k} z^{m}=\frac{1}{2}\left[\sum_{m=0}^{\infty} u_{m k} z^{m}+\sum_{m=0}^{\infty} \bar{u}_{k m} z^{m}\right]=\frac{1}{2}\left[\sum_{m=0}^{\infty} u_{m k} z^{m}+\left(\overline{\sum_{m=0}^{\infty} u_{k m} \bar{z}^{m}}\right)\right]
$$

or finally

$$
\sum_{m=0}^{\infty} D_{m k} z^{m}=\frac{1}{2}\left[u_{k}(z)+\left[\bar{v}_{k}(\bar{z})\right]\right]
$$

From this and the two lemmas proved above the proof of the theorem follows.

Remark. If we continue the real solution $U(z, \bar{z})$ to complex values of $x$ and $y$, i.e. if we replace $\bar{z}$ by $z^{*}$, then to every singularity at the point $z_{0}$ corresponds a plane-singularity $z=z_{0}$ in the four dimensional $\left(z, z^{*}\right)$ space.

4. Some properties of coefficients of function elements around different centers. Again let the real solution be $U(z, \bar{z})$, which we shall denote by $U_{0}(z, \bar{z})=$ $\sum_{m, k=0}^{\infty} D_{m k}^{(0)} z^{m} \bar{z}^{k}$. Suppose that $z=p \neq 0$ is a point in the circle of convergence of the series $\sum_{m=0}^{\infty} D_{m 0}^{(0)} z^{m}$. Developing $U_{0}(z, \bar{z})$ in a series of the form

$$
\sum_{m, k=0}^{\infty} D_{m k}^{(p)}(z-p)^{m}(\bar{z}-\bar{p})^{k}, D_{m k}^{(p)}=\left(\overline{D_{k m}^{(p)}}\right),
$$

we have in the common domain of regularity

$$
\begin{gathered}
U_{0}(z, \bar{z})=\sum D_{m k}^{(p)}(z-p)^{m}(\bar{z}-\bar{p})^{k} \equiv U_{p}(\xi, \bar{\xi}), \\
\xi=z-p, \quad \bar{\xi}=\bar{z}-\bar{p} .
\end{gathered}
$$

The relation between the subsequence $\left\{D_{m 0}^{(0)}\right\}$ and $\left\{D_{m 0}^{(p)}\right\}$ is established in using the integral operator. (See (2.6)).

We have (see [1])

$$
\begin{aligned}
& U_{0}(z, \bar{z}) \\
& \quad=\operatorname{Re}\left[R_{0}\left(z, z^{*}\right)\left[g_{0}(z)+\sum_{n=1}^{\infty} \frac{Q_{0}(n)\left(z, z^{*}\right)}{2^{2 n} B(n, n+1)} \int_{0}^{z}(z-\zeta)^{n-1} g_{0}(\zeta) d \zeta\right]\right] \\
& U_{p}(\xi, \bar{\xi}) \\
& \quad=\operatorname{Re}\left[R_{p}(\xi, \bar{\xi})\left[g_{p}(\xi)+\sum_{n=1}^{\infty} \frac{Q_{p}(n)(\xi, \bar{\xi})}{2^{2 n} B(n, n+1)} \int_{0}^{\xi}(\xi-\zeta)^{n-1} g_{p}(\zeta) d \zeta\right]\right] .
\end{aligned}
$$

From (4.2) follows

$$
U_{0}(z, 0)=\frac{1}{2}\left[g_{0}(z)+R^{*}(0, z) g_{0}^{*}(0)\right], \quad U_{p}(\xi, 0)=\frac{1}{2}\left[g_{p}(\xi)+R_{p}^{*}(0, \xi) g_{p}^{*}(0)\right] .
$$


Denoting now $\overline{\lim }_{m=\infty} \sqrt{\left|D_{m 0}^{(0)}\right|}=1 / R_{0}$ and $\varlimsup_{m=\infty} \sqrt{\left|D_{m 0}^{(p)}\right|}=1 / R_{p}$, we obtain from (4.3) and (4.1), by using again Bergman's theorem mentioned above (see [3]), the following relation:

$$
R_{p} \geqq R_{0}-|p| \text {. }
$$

We wish to add that this relation is independent of the coefficients of (1.1).

\section{BIBLIOGRAPHY}

[1] Stefan Bergman, Zur Theorie der Funktionen, die eine lineare partielle Differtialgleichung befriedigen, Matematiceski Sbornik, vol. 44 (1937), pp. 1169-1198.

[2] Stefan Bergman, Linear operators in the theory of partial differential equations, Transactions of the American Math. Soc., vol. 53 (1953), pp. 130-155.

[3] Stefan Bergman, Certain classes of analytic functions of two real variables and their properties, Trans. of the American Math. Soc., vol. 57 (1945), pp. 299-331. 